\title{
UNA EXPERIENCIA DE EVALUACIÓN FORMATIVA EN EL GRADO EN CIENCIAS DE LA ACTIVIDAD FÍSICA Y EL DEPORTE: UTILIZACIÓN DE RÚBRICAS Y FEEDACK PARA LA MEJORA DE LOS APRENDIZAJES
}

A formative assessment experience in Physical Activity and Sport Sciences Degree: Implementation of rubrics and feedback from improvement of learning

Uma experiência de avaliação formativa na Licenciatura em Ciências da Atividade Física e Desporto: utilização de rubricas e feedback para a melhoria das aprendizagens

\section{Laura Cañadas}

Departamento de Educación Física, Deporte y Motricidad Humana. Universidad Autónoma de Madrid, España. Teléfono: +34 4975924. Correo electrónico: laura.cannadas@,uam.es. ORCID: https://orcid.org/0000-0003-4179-9018

\section{Resumen}

En este trabajo se presenta una experiencia de evaluación formativa donde se fomenta la regulación del trabajo a partir de unos criterios e instrumentos de evaluación conocidos por el alumnado y la utilización de un feedback continuo. Se desarrolló con 40 estudiantes en el Grado en Ciencias de la Actividad Física y el Deporte. La experiencia de evaluación formativa se aplicó en una tarea donde el alumnado recogía los contenidos trabajados en la sesión práctica, la reflexión sobre la idoneidad de lo trabajado y propuestas alternativas. Se les facilitaba la descripción de la tarea a realizar, la rúbrica de evaluación y feedback de carácter cualitativo individual tras la entrega de cada una de las tareas con el objetivo de ir mejorando el desempeño en las siguientes. Se valoró la utilidad de la experiencia para la regulación del aprendizaje del alumnado y la mejora de los procesos de aprendizaje.

Palabras clave: Evaluación formativa; feedback; regulación; rúbrica

\begin{abstract}
This paper presents a formative assessment experience where the regulation of work is encouraged based on criteria and assessment tools known by the students and the use of continuous feedback. It has been developed with 40 students in the Degree in Physical Activity and Sport Sciences. The experience was carried out in a task where the students had to collect the contents worked in the session given by the teacher, the reflection on the suitability of the work and alternative proposals. They were given the description of


the task, the assessment rubric and individual qualitative feedback after the delivery of each of the tasks with the aim of improving performance in the following ones. The usefulness of the experience for the regulation of student learning and the improvement of learning processes was valued.

Keywords: Formative assessment; feedback; regulation; rubric

\section{Resumo}

Este trabalho apresenta uma experiência de avaliação formativa onde se fomenta a regulação do trabalho a partir de critérios e instrumentos de avaliação conhecidos pelos alunos e o uso de feedback contínuo. Foi desenvolvida com 40 alunos da Licenciatura em Ciências da Atividade Física e Desporto. A experiência de avaliação formativa foi aplicada numa tarefa em que os alunos recorriam aos conteúdos trabalhados na sessão prática, à reflexão sobre a adequação do trabalho e a propostas alternativas. Eles receberam a descrição da tarefa a ser realizada, os critérios de avaliação e feedback de caráter qualitativo individual, após a entrega de cada uma das tarefas, com o objetivo de melhorar o desempenho nas seguintes. A experiência foi útil para a regulação da aprendizagem dos alunos e melhoria dos processos de aprendizagem.

Palavras-chave: Avaliação formativa; feedback; regulação; rubrica

\section{Introducción}

El empleo de la evaluación formativa en la formación universitaria está cada vez más extendido (López-Pastor \& Sicilia-Camacho, 2017). Su empleo ha mostrado mejoras en el desarrollo de las competencias del alumnado (Hortigüela, Palacios \& López-Pastor, 2018). Para que esto pueda ser así, las investigaciones han mostrado que hay una serie de factores que deben estar presentes en las propuestas de evaluación formativa (Cañadas, 2018): (a) sistemática y programada de antemano; (b) con criterios de evaluación conocidos por todos los agentes implicados; (c) realizarse en diferentes momentos del proceso; (d) proporcionar feedback; (e) fomentar la participación del alumnado; y, (f) la utilización de diferentes instrumentos de evaluación.

Así, en este artículo, presentamos una experiencia de evaluación formativa donde se implican la mayoría de estos indicadores. Esta experiencia busca la regulación del trabajo por parte del propio estudiante utilizando para ello unos instrumentos y criterios 
de evaluación conocidos por todo el alumnado desde el comienzo de curso y la utilización de un feedback continuo

\section{Contextualización}

La experiencia se ha llevado a cabo en la titulación de Ciencias de la Actividad Física y el Deporte (CCAFyD), en la materia de Deportes Colectivos y su Didáctica III: Voleibol y Rugby ( $2^{\circ}$ Curso - 6 ECTS $)$ en la Universidad Autónoma de Madrid. De las 150 horas de la asignatura, 68 serán de carácter presencial y 82 de carácter no presencial. En la asignatura se encuentran matriculados 40 estudiantes.

\section{Diseño y desarrollo}

Para el diseño de la experiencia de evaluación se partió de lo recogido en la guía docente respecto a qué tipo de actividades debe realizar el alumnado y cómo, de manera global, debe ser la evaluación. Las tareas de evaluación y calificación de la materia deben agruparse en: (a) un 70\% de la calificación final destinada al trabajo continuo y; (b) un 30\% destinado a una prueba de carácter escrito al final de la asignatura.

Partiendo de esta organización, se estableció un sistema concreto para la evaluación de los aprendizajes del alumnado. Este consistía en:

1) Tarea que recoge los contenidos trabajados en la sesión impartida por el docente, así como la reflexión sobre la idoneidad de lo trabajado y propuestas alternativas (40\% de la calificación).

2) La programación y puesta en práctica de una sesión de enseñanza y aprendizaje sobre ese deporte, y la reflexión sobre la puesta en práctica $(30 \%$ de la calificación).

3) Prueba escrita al final del cuatrimestre (30\% de la calificación).

Aquí se presenta lo relacionado con la primera de las tareas de evaluación. Esta se planteaba con un doble objetivo, por una parte, que al finalizar la asignatura tengan un conjunto de sesiones relacionadas con cada uno de los deportes, y por otro, que aprendan a ser críticos con los procesos de enseñanza y aprendizaje, y sepan plantear alternativas concretas para lograr los objetivos de aprendizaje de cada uno de los deportes que componen la materia. La entrega debía realizarse 4 días después de la sesión, y se devolvía con retroalimentación sobre el grado de desempeño en la misma y 
cómo mejorarla como muy tarde 24 horas después de la entrega. En total se debían entregar 6 tareas de cada deporte.

El alumnado recibía una triple información: (a) la propia descripción de la tarea a realizar, donde se explicaba el objetivo de esta y los apartados que debía contener; (b) se les entregaba una rúbrica con los aspectos que se iban a valorar y los posibles grados de calidad en cada parte de la tarea; y, (c) en cada una de las tareas se proporcionaba feedback cualitativo en cada uno de los apartados para su posterior mejora, además de indicarles cuál era su grado de desempeño en la rúbrica. La primera de las tareas que debía entregarse se evaluaba por parte del profesorado devolviéndoselo al alumnado con feedback, pero esta tarea no era calificada, con objeto de que el alumnado tuviese desde el principio la información completa sobre cómo realizar las tareas.

\section{Evaluación}

La valoración de la experiencia se realizará desde el punto de vista del profesorado a través de las ventajas e inconvenientes detectados:

a. Ventajas: Encontramos una mejora en la realización de las tareas y, especialmente en la capacidad para ser críticos y realizar propuestas a los procesos de enseñanza y aprendizaje. Así mismo, el interés del alumnado por cómo mejorar las tareas, y, por consiguiente, estas mejoraron. Por otra parte, la posibilidad de recibir información concreta respecto al desempeño en el trabajo en relación con aquello que se esperaba que el alumnado fuese capaz de hacer en las tareas (rúbrica). La posibilidad del alumnado de autorregular sus tareas tomando como referencia los criterios de evaluación establecidos en la rúbrica

b. Inconvenientes: la cercanía en el tiempo de ambas entregas hacía que en ocasiones el alumnado no realizase de manera adecuada alguna de las tareas. La carga para el profesorado, corrigiendo semanalmente 80 trabajos durante 6 semanas.

\section{Conclusiones}

La valoración de la experiencia tras este primer año de implantación ha sido positiva valorando su utilidad para la regulación del aprendizaje del alumnado y la mejora de los procesos de aprendizaje. El alumnado ha podido saber desde el primer momento con qué criterios se le iba a evaluar y calificar, pudiendo autoevaluar la calidad de sus 
tareas, de la misma forma que ha recibido información continua y con carácter inmediato para poder mejorar su trabajo, y por tanto su aprendizaje. Para futuras experiencias sería necesario espaciar más las entregas para que tanto la carga de trabajo del docente como del alumnado sea más equilibrada.

\section{Referencias}

Cañadas, L. (2018). La evaluación formativa en la adquisición de competencias docentes en la formación inicial del profesorado de Educación Física. Tesis Doctoral Inédita. Madrid: Universidad Autónoma de Madrid.

Hortigüela, D., Palacios, A., \& López-Pastor, V.M. (2018). The impact of formative and shared or co-assessment on the acquisition of transversal competences in higher education. Assessment \& Evaluation in Higher Education, 44(6), 933-945. DOI: $\underline{10.1080 / 02602938.2018 .1530341}$

López-Pastor, V.M., \& Sicilia-Camacho, A. (2017). Formative and shared assessment in higher education. Lessons learned and challenges for the future. Assessment \& $\begin{array}{lllll}\text { Evaluation in Higher Education, 42(1), } & \text { 77-97. }\end{array}$ DOI:10.1080/02602938.2015.1083535 